\title{
MULTIPLICATIVE FIBRE MAPS
}

\author{
BY LARRY SMITH ${ }^{1}$
}

Communicated by John Milnor, January 9, 1967

In this note we shall outline a result concerning the cohomology of a multiplicative fibre map. To fix our notation we shall assume that

$$
F \stackrel{i}{\rightarrow} E \stackrel{\pi}{\rightarrow} B
$$

is a Serre fibre space such that

(1) $F, E, B$ are $H$-spaces (homotopy associative) and $F \rightarrow E, E \rightarrow B$ are $H$-maps.

(2) $B$ is simply connected.

(3) $H^{*}\left(B ; Z_{p}\right)$ is a polynomial algebra, where $Z_{p}$ denotes the integers modulo $p, p$ a prime.

(4) $H_{*}\left(B ; Z_{p}\right)$ is a commutative algebra. The result that we shall establish is

THEOREM. If $H^{*}\left(E ; Z_{p}\right)$ and $H^{*}\left(B ; Z_{p}\right)$ are of finite type and $p$ is an odd prime, then

$$
\left.H^{*}\left(F ; Z_{p}\right) \cong \operatorname{Tor}_{H^{*}(B ;} ; Z_{p}\right)\left(Z_{p}, H^{*}\left(E ; Z_{p}\right)\right)
$$

as an algebra over $Z_{p}$. (A similar result holds over the rationals $Q$.)

The result for $p=2$ is more complicated to state and is treated in Theorem 3.

In fact, as we shall see, we can compute the indicated torsion product simply from a knowledge of the cohomology map

$$
\pi^{*}: H^{*}\left(B ; Z_{p}\right) \rightarrow H^{*}\left(E ; Z_{p}\right) .
$$

Results and techniques similar to these have been used in [8] to compute the $Z_{p}$-cohomology of stable two stage Postnikov systems.

This announcement serves as an introduction to the joint work of J. C. Moore and the author that will appear elsewhere.

1. Algebra. Throughout this section $k$ will denote a fixed field and $\otimes$ will mean $\otimes_{k}$. We shall assume that the reader is familiar with the material covered in the homological algebra section of [1]. All mod ules are assumed of finite type. All algebras will be assumed graded augmented and connected.

Definition. If $\Gamma$ is a Hopf algebra over $k$, an ideal $I \subset \Gamma$ is called

\footnotetext{
1 Partially supported by NSF-GP-3946.
} 
a Hopf ideal, iff

$$
\nabla(I) \subset \Gamma \otimes I+I \otimes \Gamma
$$

where $\nabla: \Gamma \rightarrow \Gamma \otimes \Gamma$ is the coproduct in $\Gamma$.

Proposition 1. If $\Gamma$ is a commutative and cocommutative Hopf algebra over $k, I \subset \Gamma$ is a Hopf ideal, then there exists a unique sub-Hopf algebra $\Lambda \subset \Gamma$ such that $I=\bar{\Lambda} \circ \Gamma .(\bar{\Lambda}=\operatorname{ker} \Lambda \rightarrow k$.

Proof. Let $\Omega=\Gamma / I$. Then $\Omega$ is a Hopf algebra in a natural way and the natural map

$$
\nu: \Gamma \rightarrow \Omega
$$

is an epimorphism of Hopf algebras. Passing to duals we obtain a monomorphism of Hopf algebras

$$
\nu^{*}: \Omega^{*} \rightarrow \Gamma^{*} \text {. }
$$

Since $\Gamma^{*}$ is commutative we can set $A=\Gamma^{*} / / \Omega^{*}$. Passing to duals again and identifying $\Gamma$ with its double dual we obtain $A^{*} C \Gamma$. If we set $\Lambda=A^{*}$ it is straight forward to verify that $\Lambda$ has the required properties (see for example Proposition 4.4 of [7]).

Notation. Let $\Gamma, A$ be commutative and cocommutative Hopf algebras over $k, \phi: \Gamma \rightarrow A$ a map of Hopf algebras. Then $\operatorname{ker} \phi \subset \Gamma$ is a Hopf ideal. Hence by Proposition 1 there is a sub-Hopf algebra $\Lambda$ of $\Gamma$ with $\bar{\Lambda} \circ \Gamma=\operatorname{ker} \phi$. We will adopt the notation subker $\phi$ for $\Lambda$.

Proposition 2. Suppose that $\Gamma$ is a commutative, cocommutative Hopf algebra over $k, A$ is a Hopf algebra over $k$ and $\phi: \Gamma \rightarrow A$ is a map of Hopf algebras, then

$$
\operatorname{Tor}_{\Gamma}(k, A) \cong A / / \phi \otimes \operatorname{Tor}_{\text {subker } \phi}(k, k)
$$

as an algebra.

Proof. According to $[7,4.4] \Gamma$ is a free subker $\phi$-module. Hence by $\left[2\right.$, Theorem $6.1 \mathrm{p}$. 349] we have a spectral sequence $\left\{E_{r}, d_{r}\right\}$ such that

$$
E_{r} \Rightarrow \operatorname{Tor}_{\Gamma}(k, A),
$$

and if $\Omega=\Gamma / / \phi$

$$
E_{2}=\operatorname{Tor}_{\Omega}\left(\operatorname{Tor}_{\text {subker } \phi}(k, k), A\right) .
$$

But $\Omega \subset A$ is a sub-Hopf algebra, hence by $[7,4.4]$ again, $A$ is a free $\Omega$-module. Therefore the edge homomorphism of the spectral sequence provides an isomorphism 


$$
A \otimes_{\mathbf{\Omega}} \operatorname{Tor}_{\mathrm{subker} \phi}(k, k) \cong \operatorname{Tor}_{\Gamma}(k, A) .
$$

Finally as in $[1, \S 2.3]$ one can show that $\operatorname{Tor}_{\text {subker } \phi}(k, k)$ is a trivial $\Omega$-module, and hence

$$
A \otimes \Omega \operatorname{Tor}_{\text {subker } \phi}(k, k) \cong A / / \Omega \otimes \operatorname{Tor}_{\text {subker } \phi}(k, k)
$$

and the result follows.

Notation. We shall adopt the notation $P\left[x_{1}, \cdots, x_{n}, \cdots\right]$ for a graded polynomial algebra over $k$ on generators $x_{1}, \ldots$ of degree $\operatorname{deg} x_{1}, \cdots$.

Similarly $E\left[y_{1}, \ldots\right]$ will denote a graded exterior algebra on generators $y_{1}, \cdots$.

We note that if the characteristic of $k$ is not 2 then $\operatorname{deg} x_{1}, \cdots$ are all even.

We are now ready to make our main calculation. We therefore make the following assumptions:

(1) $k=Z_{p}, p$ any prime or $k=Q$ the rational numbers.

(2) $\Gamma$ is a Hopf algebra over $k$

(a) As an algebra $\Gamma \cong P\left[x_{1}, \cdots\right]$.

(b) As a coalgebra $\Gamma$ is commutative.

(3) $A$ is a Hopf algebra over $k$ and $\phi: \Gamma \rightarrow A$ is a map of Hopf algebras.

Main Calculation. Under the above conditions

where

$$
\operatorname{Tor}_{\Gamma}(A, k) \cong A / / \phi \otimes E\left[u_{1}, \cdots\right]
$$

$$
u_{i} \in \operatorname{Tor}_{\Gamma}^{1, *}(A, k) \quad i=1, \cdots .
$$

Proof. By Proposition 2,

$$
\operatorname{Tor}_{\mathrm{r}}(A, k) \cong A / / \phi \otimes \operatorname{Tor}_{\text {subker } \phi}(k, k) \text {. }
$$

By construction subker $\phi \subset \Gamma$ is a sub-Hopf algebra. By Borel's structure theorem for Hopf algebras over $k[7,7.11]$ subker $\phi \cong P\left[v_{1}, \cdots\right]$ and the result now follows by the graded version of $[6$, Theorem 2.2 , p. 205].

2. Multiplicative fibre maps. Suppose that $F \rightarrow E \rightarrow B$ is a Serre fibre space, $B$ simply connected and all cohomology in sight is of finite type.

THEOREM (EILENBERG-MOORE [3]). There exists a second quadrant spectral sequence $\left\{E_{r}, d_{r}\right\}$ such that

(1) $E_{r} \Rightarrow H^{*}(F ; k)$. 
(2) $E_{2}^{p, q}=\operatorname{Tor}_{H *(B ; k)}^{-p, q}\left(k, H^{*}(E ; k)\right), \quad p \leqq 0$.

(3) $E_{r}$ is in a natural way an algebra and $d_{r}$ is a derivation of degree $(r, 1-r)$.

Theorem 3. Suppose that

$$
F \rightarrow E \stackrel{\pi}{\rightarrow} B
$$

is a multiplicative fibre map over the simply connected base space B. In addition assume that $k=Z_{p}, p$ any prime or $k=Q$ and

(1) $H^{*}(B ; k)=P\left[x_{1}, \cdots\right]$.

(2) $H_{*}(B ; k)$ is commutative.

Let $\left\{E_{r}, d_{r}\right\}$ denote the Eilenberg-Moore spectral of

$$
F \rightarrow E \stackrel{\pi}{\rightarrow} B
$$

Then

(1) $E_{2} \cong H^{*}(E ; k) / / \pi^{*} \otimes E\left[u_{1}, \cdots\right]$ as an algebra, where $u_{i} \in E_{2}^{-1, *}$ $i=1, \cdots$.

(2) $E_{2}=E_{\infty}=E^{0} H^{*}(F ; k)$.

ProOF. (1) follows directly from the main calculation of the first section. To see (2) observe that

$$
d_{r}\left(E_{2}^{-p, *}\right) \subset E_{2}^{-p+r, *}=0 \text { if } p=0,1 \text { and } r \geqq 2 .
$$

Hence $d_{r}$ vanishes on the algebra generators of $E_{2}$ and since it is a derivation we must have $d_{r}=0, r \geqq 2$.

CoRollary 4. If $k=Z_{p}$, $p$ an odd prime, or $k=Q$, then under the hypotheses of Theorem 3 there is an isomorphism of algebras

$$
H^{*}(F ; k) \cong \operatorname{Tor}_{H^{*}(B ; k)}\left(k, H^{*}(E ; k)\right) \text {. }
$$

Proof. One merely notes that for a suitable filtration

$$
\begin{aligned}
E^{0} H^{*}(F, k) & \cong H^{*}(E ; k) / / \pi^{*} \otimes E\left[u_{1}, \cdots\right] \\
& \cong \operatorname{Tor}_{H^{*}(B ; k)}\left(k, H^{*}(E ; k)\right)
\end{aligned}
$$

and that $E\left[u_{1}, \cdots\right]$ is a free commutative algebra. The result now follows by standard arguments.

REMARK 1 . Theorem 3 can be used to calculate the $Z_{2}$-cohomology of stable two-stage Postnikov systems. (See [4], [5], [8].) It can also be used to simplify somewhat the calculations of [9]. From these calculations one can obtain the $Z_{2}$ cohomology of the stages in the Postnikov tower of SO. 
REMARK 2. It may be of interest, when more results are in, to apply Corollary 4 to the fibration

$$
P L \rightarrow F \rightarrow F / P L .
$$

\section{REFERENCES}

1. J. F. Adams, Hopf invariant one, Ann. of Math. 72 (1960), 20-104.

2. H. Cartan and S. Eilenberg, Homological algebra, Princeton Univ. Press, Princeton, N. J., 1956.

3. S. Eilenberg and J. C. Moore, Homology and fibrations. I, II, III, ‥, Comment. Math. Helv. (to appear).

4. L. Kristensen, Cohomology of spaces with two non vanishing homotopy groups, Math. Scand. 12 (1963), 83-105.

5. W. S. Massey and F. P. Peterson, Cohomology of two stage Postnikov systems, (to appear).

6. S. MacLane, Homology, Springer-Verlag, Berlin; Academic Press, New York 1963.

7. J. Milnor and J. C. Moore, Structure of Hopf algebras, Ann. of Math. 81 (1965), 211-264.

8. L. Smith, Cohomology of two stage Postnikov systems, Illinois J. Math. (to appear).

9. R. Stong, Determination of $H^{*}\left(B O(k, \cdots, \infty) ; Z_{2}\right)$ and $H^{*}\left(B U(k, \cdots, \infty) ; Z_{2}\right)$, Trans. Amer. Math. Soc. 107 (1963), 526-544.

Princeton University 